\title{
Ellipsometric Analysis of Cadmium Telluride Films' Structure
}

\author{
Anna Evmenova, ${ }^{1}$ Volodymyr Odarych, ${ }^{2}$ Mykola Vuichyk, ${ }^{1}$ and Fedir Sizov ${ }^{1}$ \\ ${ }^{1}$ V. Lashkaryov Institute of Semiconductor Physics, NAS of Ukraine, 41 Prospect Nauky, Kyiv 03028, Ukraine \\ ${ }^{2}$ Taras Shevchenko Kyiv National University, Physics Department, 2 Prospect Academician Glushkov, Kyiv 03022, Ukraine
}

Correspondence should be addressed to Fedir Sizov; sizov@isp.kiev.ua

Received 5 January 2015; Revised 5 April 2015; Accepted 6 April 2015

Academic Editor: Lian Gao

Copyright (c) 2015 Anna Evmenova et al. This is an open access article distributed under the Creative Commons Attribution License, which permits unrestricted use, distribution, and reproduction in any medium, provided the original work is properly cited.

\begin{abstract}
Ellipsometric analysis of CdTe films grown on Si and CdHgTe substrates at the "hot-wall" epitaxy vacuum setup has been performed. It has been found that ellipsometric data calculation carried out by using a simple one-layer film model leads to radical distortion of optical constants spectra: this fact authenticates the necessity to attract a more complicated model that should include heterogeneity of films. Ellipsometric data calculation within a two-layer film model permitted to conclude that cadmium telluride films have an outer layer that consists of the three-component mixture of CdTe, cavities, and basic matter oxide. Ratio of mixture components depends on the time of deposition, that is, on the film thickness. The inner layer consists of cadmium telluride.
\end{abstract}

\section{Introduction}

Semiconductor films are widely used in various sectors of micro- and optoelectronics for construction of signal transmission devices; thin film electroluminescent devices based on cadmium telluride are used as emitters; CdTe films serve as protecting coatings on the surface of luminous energy receivers in the IR spectral range. Solar energy sensors based on CdTe manufactured up to date are among the most efficient ones (http://solarlove.org/top-solar-module-manufacturers-2013/) (First Solar Company).

The following factors are to be known for designing film devices: optical constants and thickness of the films, area distribution of film thickness as well as radiation and chemical stability of film structures.

The "hot-wall" epitaxy vacuum method used in up-todate study of film properties allows obtaining qualitative films, in which thickness is controlled by time of sputtering. For growing films that could be used as a base for device construction, the time of optimal deposition equals 6 to $8 \mathrm{~min}$ utes. For this period, hard-grained films with a monocrystalline or polycrystalline structure subject to substrate and technological conditions of sputtering, temperature conditions in reaction chamber specifically, are generated.
According to AFM data, there is more or less developed relief on the surface of the films grown using the vacuum "hot-wall" epitaxy method; this fact indicates availability of a rather complex film structure.

Reflective ellipsometry, which in some cases is rather sensitive to reflective system characteristics and permits determination of its parameters with sufficiently high accuracy, is the efficient method for studying the film structure and properties.

There are papers where properties of a cadmium telluride films are studied using the ellipsometric method. In [1-7] and also in our papers $[8,9]$, it is shown that the surface structure and film optical constant values strongly depend on the methods and conditions of film deposition. Specifically, film optical constants, refractive index $n$ and index of extinction $\kappa$, appeared to differ perceptibly from optical parameters of monocrystalline cadmium telluride. Particularly, the refractive index of the films is always smaller than that of monocrystalline cadmium telluride. In [6] this fact is explained by film porosity; and in [2,5] high sensitivity of film optical properties to composition deviation from stoichiometry is registered in the film near-surface layer. Formation of the hexagonal phase in composition of the films with a high thickness [3] or in films that were grown at high 
temperature of substrate $[1]$ is observed. It is found in $[8,10]$ that the refractive index in the visible light long-wave region increases with film thickness rising.

However, the index of extinction could be both lower and higher than that of CdTe [5], which could not be explained by availability of pores in the film.

The one-layer uniform film model is mainly used for processing ellipsometric data. At the same time, we need to choose more complex reflecting system model of tested objects for ellipsometric data interpretation taking into account technological conditions of film production as well as optical study and atomic force microscopy experimental data analysis. Noticeable distortion of system parameters obtained during data processing could appear when using the model that contradicts to the fact.

Existence of a relief could force a researcher to choose a more complicated film model than the single-layer one. At the same time it is known that ellipsometric data sensitivity to the thin details of the reflective system structure is the highest in the fundamental absorption range of semiconductors, which is caused by intrinsic electron transitions between energy bands.

The aim of this paper is to find such system model that would be sufficiently simple and able to describe in the best way ellipsometric data obtained at light reflection from the real cadmium telluride film surface and to determine its parameters.

To achieve the aim, we have broaden the spectral range of ellipsometric measurements and also used two-layer film model for interpretation of their data.

\section{Materials and Methods}

Epitaxial thin CdTe films were grown by the vacuum "hotwall" epitaxy method. Monocrystalline silicon plates and cadmium-mercury-telluride $\mathrm{Cd}_{0.2} \mathrm{Hg}_{0.8}$ Te plates, which lattice constant approximates to the cadmium telluride one, were used as substrates. Before loading into the setting, the substrates were washed in HF acid and then in acetone for removing oxides and contaminations. Formation of $\mathrm{CdTe}$ films was performed in high vacuum $\sim 10^{-7}$ Torr. Temperature parameter values that qualified conditions of thin CdTe films growth were as follows: source temperature was $T_{\text {source }} \approx$ $380^{\circ} \mathrm{C}$, "hot" wall temperature was $T_{\text {wall }} \approx 400^{\circ} \mathrm{C}$, and substrate temperature was $T_{\text {sub }} \approx 50 \cdots 80^{\circ} \mathrm{C}$. Rather low substrate temperatures are required to eliminate the process of mercury evaporation from $\mathrm{HgCdTe}$ substrates. Nominal thicknesses of epitaxial CdTe layers were obtained by thin layer continuous growth during the sputtering time. Films were grown separately for fixed times: 6,10 , and 14 minutes. In such a way, we obtained 4 series of films with different area interference color that specifies different film thicknesses at its each section. Specification of the samples is given in Table 1 .

Morphology and X-ray examinations of this type films obtained using the same device were described in [11]. According to X-ray data, all the films have the crystalline structure.
TABLE 1: Specification of the samples.

\begin{tabular}{lcccc}
\hline Substrate & Series & $\begin{array}{c}\text { Film } \\
\text { notation }\end{array}$ & $\begin{array}{c}\text { Time of } \\
\text { deposition, } \\
\text { min }\end{array}$ & $\begin{array}{c}\text { Number of } \\
\text { measured places }\end{array}$ \\
\hline \multirow{3}{*}{$\mathrm{Si}$} & I & F1 & 10 & 2 \\
& & F2 & 6 & 3 \\
& F3 & 14 & 4 \\
& II & F4 & 10 & 4 \\
& & F5 & 14 & 4 \\
\hline \multirow{3}{*}{ CdHgTe } & III & F6 & 8 & 4 \\
& & F7 & 10 & 2 \\
& & F8 & 14 & 3 \\
& IV & F9 & 10 & 3 \\
& & F10 & 14 & 3
\end{tabular}

Ellipsometric measurements were performed using the $632.8 \mathrm{~nm}$ wavelength of LEF-3M-1 compensatory zeroellipsometer, and 579, 546, 435, 405, and $366 \mathrm{~nm}$ spectral lines of mercury lamp radiation were used when measuring with spectral photometric ellipsometer. The measured values were the ellipsometric parameter $\Delta$ (phase difference between $p$ and $s$-components of reflected light wave electric vector) and $\psi$ (arctangent of the ratio between refractive indexes in $p$ - and $s$-planes of the sample), for the photometric method $-\cos \Delta$ and $\operatorname{tg} \psi$.

2.1. One-Layer Model Calculation. Results are given as points on the diagram of measured values $\cos \Delta$ and $\operatorname{tg} \psi$. At the first stage of estimations, film parameters were calculated using the measured ellipsometric parameters considering the film to be homogenous in physical properties along its thickness. In this case, the calculated parameters are the refractive index $n$, index of extinction $\kappa$, and film thickness $d$ in the measured area.

The designed earlier Method of Thickness Curves (further MTC) [12] based on the software package "Ellipsometric calculator" [13] was used for optical constants and film thickness estimation. Values for substrates' refractive index and index of extinction were taken from literary data, for monocrystalline silicon from [14] and for CdHgTe of the same composition from [15].

MTC permits determination of optical constants at different places of the same sample; these places have equal optical parameters but different film thicknesses. At the first stage, using the pair of ellipsometric parameters, obtained at each place, values array of film optical constants $n$ and $\kappa$ was calculated. Calculation was executed for several values of thickness in the range of expected value. This data array looks like a curve (thickness curve) on the diagram in $n$, $\kappa$ coordinates; the film thickness $d$ changes lengthwise this curve. The curve number is equal to the number of studied places.

Each thickness curve has a physical meaning of values array of studied system parameters in the chosen place of sample surface, which fit ellipsometric parameters measured 


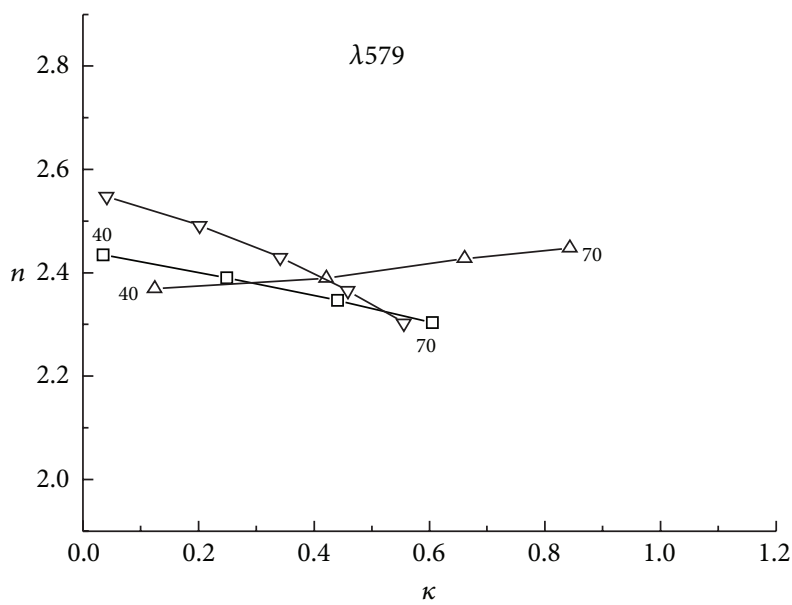

(a)

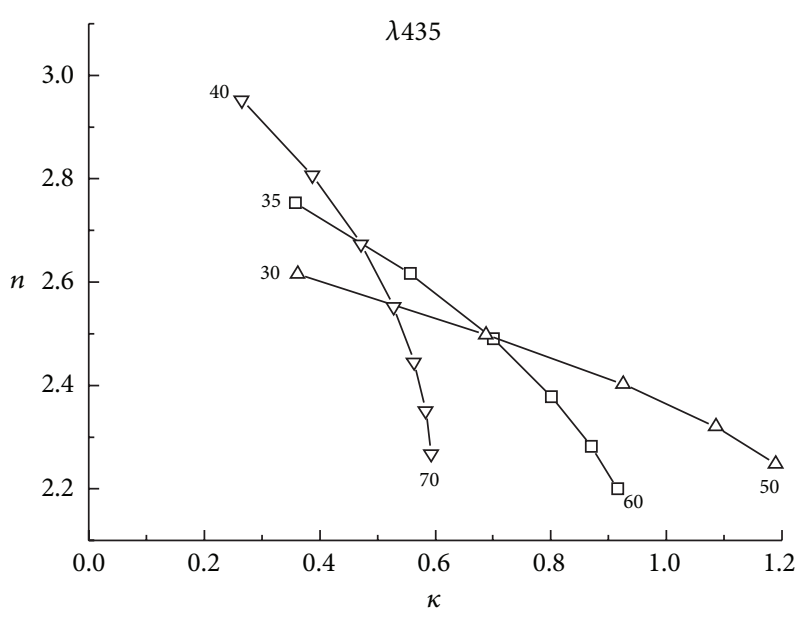

Places
$-\square-1$
$-\triangle-2$
$-\nabla-3$

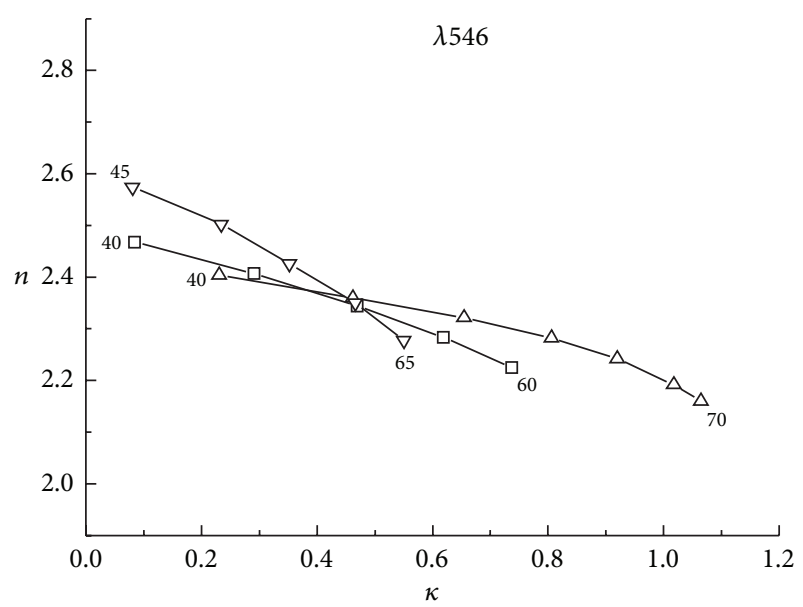

(b)

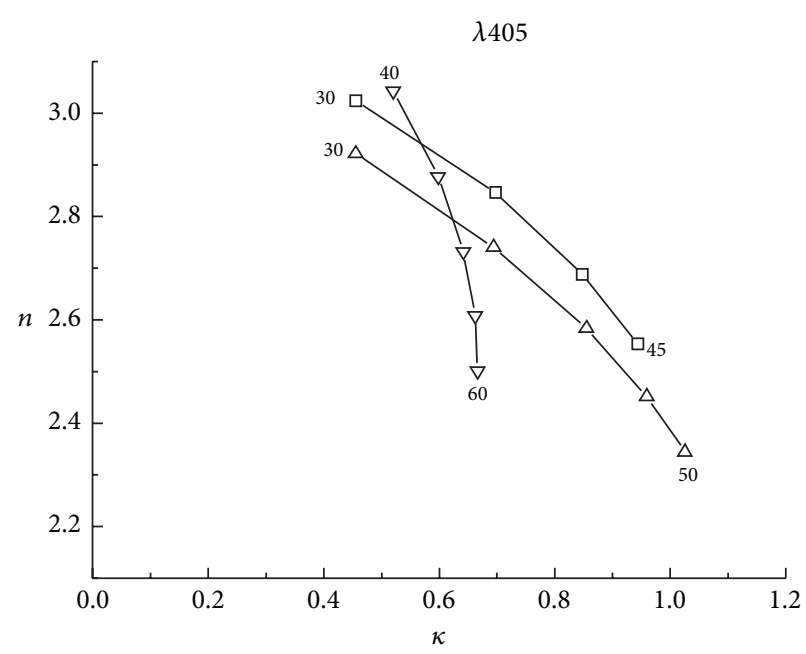

Places

$-\square-1$

$-\triangle-2$

$-\nabla-3$

(c)

(d)

FIGURE 1: Range of values of refractive index $n$ and index of extinction $\kappa$ (curves), calculated using experimental data for different places of the film F2 and different wavelengths $\lambda(\mathrm{nm})$. The numbers near markers specify the film thickness in $\mathrm{nm}$.

at this place. If the film is homogenous over the whole surface, all the thickness curves, probable values arrays, will intercross in a single point. Because of errors, they will intercross in some area, the area of approximate convergence of the curves, which specifies required film optical parameters' values.

Use of this method for the homogenous film model ascertained some peculiarities in the process of system parameters determination.

The first peculiarity concerns law of thickness curves transformation with changing the wavelength. For instance, Figure 1 shows results of the film F2 parameters calculation within the one-layer model for several wavelengths. There are three work places for this film, so three thickness curves are adduced in Figure 1. They intercross in pairs and form confined areas of approximate convergence. The scale of axes is identical, so it is obvious that curves disperse with wavelength decreasing; the intercross area increases and the confined area does not exist for the wavelength $405 \mathrm{~nm}$.

Similar results were obtained for all the studied films; the shorter the wavelength is, the more obvious thickness curves' dispersion is. Almost for all the films there is the shortest wavelength, starting with which the confined area of thickness curves intercross does not exist; that is why all three film parameters could not be found simultaneously. Optical constants at these wavelengths were determined using the film thickness for the same places that were found in the longwave spectral range.

In the frames of this one-layer model, film optical constants in the short-wave range seem to depend on thickness; that is, they are different at places with different thicknesses.

So, the size of the intercross area of thickness curves, which characterizes the range of probable values of film 
parameters, increases with decreasing the wavelength; error of determined parameters increases correspondingly.

The second conclusion is related with determination of peculiarities inherent to unknown parameters of films with different sputtering times, that is, films with different thicknesses.

As an example Figure 2 shows the results of calculating the parameters of CdTe films obtained for different sputtering times on silicon substrates.

Thickness curves, using which indeterminate parameters were found, are presented in Figure 2(a); the graphic dependence of ellipsometric parameters on the film thickness, calculated using the obtained parameters, is presented in the Figure 2(b) in combination with the experimental data.

Obviously, close intercross area of thickness curves is observed for the film F2 that has the least thickness among the studied films. Film parameters were determined using the intercross points coordinates; the theoretical curve calculated using these parameters describes well location of experimental points obtained at light reflection from film places with different thicknesses. The thickness of the film F2 changes in area extent from 40 up to $60 \mathrm{~nm}$ (Figure 2(b)).

For the film F4 grown for 10 minutes, there are two sets of thickness curves and, respectively, two solution sets denoted in Figure 2(b) by the numbers 1 and 2. The thickness curves 2 do not intercross and therefore do not give film parameters common for all the film places. The thickness curves 1 have the well confined intercross area and obtained by its position film parameters describe well experimental results (Figure 2(b)). The thickness of the film F4 changes in area extent from 60 up to $78 \mathrm{~nm}$.

For the film F5, with sputtering time of 14 minutes, two sets of curves also exist, but any single intercross area for all four thickness curves both for the first and second sets does not exist. A curve for one of the sets goes separately. So, the thickness curves for the first set describe well location of only three experimental points obtained for this sample. The thickness of film F5 changes in area extent from 120 up to $140 \mathrm{~nm}$.

Passing from the film F2 to film F5, that is, with increasing the thickness, optical parameters of films' matter change appropriately; refractive index decreases and index of extinction appreciably increases even to values $\approx 1$ in the case of film F5.

Results of our analysis concerning cadmium telluride films on the CdHgTe substrate are presented in Figure 3. The same peculiarities as for films on silicon substrates could be checked out. Particularly, two sets of thickness curves are observed for each film. Only one of them gives the possibility to determine film parameters.

Thus, the film F6 thickness changes through its area within the range $70 \cdots 90 \mathrm{~nm}$, the film F9 thickness locates within the range of $94 \cdots 105 \mathrm{~nm}$, and the film F10 thickness locates within the range of $90 \cdots 150 \mathrm{~nm}$.

The index of extinction increases appreciably with film thickness growth; its value amounts to $\kappa \sim 0.9$ (film F10), which exceeds several times the value of index of extinction for monocrystalline cadmium telluride in the bulk state.
With film increasing the thickness, thickness curves spread away, and film parameters determination in the shortwave range with a sufficient accuracy is often impossible (Figure 3, film F10).

It is obvious that only second solutions, from two possible ones, describe location of the experimental points.

Thus with increasing the film sputtering time, the area of curves relative intercross expands, and it does not occur beginning with some wavelength; so all film parameters determination with a sufficient accuracy is impossible. In addition, the index of extinction values can appreciably exceed the index of extinction for bulk matter.

The described analysis was performed for all the films and at various wavelengths from visible and near ultraviolet spectral ranges. Figure 4 shows typical spectra of optical constants for one of the films in comparison with literary data [16] for monocrystalline cadmium telluride.

From the data presented in Figure 4, it is obvious that the spectra of optical constants are rebuilt with increasing the deposition time, and corresponding film thickness growth. Particularly, the maximum of extinction index, which for monocrystalline cadmium telluride is observed near $\lambda \sim 400 \mathrm{~nm}$, shifts approximately to $\lambda \sim 550 \mathrm{~nm}$, when the film thickness is higher than $75 \mathrm{~nm}$. In conformity with it, the absorption band also shifts to the long-wave spectral range.

Similar distorted spectra of optical constants were obtained for all other films.

The spectra for films with thicknesses less than $70 \mathrm{~nm}$, studied both in this paper and in our earlier publications $[8,9]$, practically match by main maximum locations with the spectra for bulk monocrystalline cadmium telluride. They differ only by absolute values of film optical parameters that are less than optical constants for monocrystalline cadmium telluride.

As these peculiarities for spectra calculated using the simple one-layer model are not meaningful, so the study should be broadened by attracting more complicated model than the one-layer one.

2.2. Two-Layer Model Calculation. Distortion of the optical constants spectrum detected from the experimental data in simple one-layer system suggests cadmium telluride films to be actually heterogeneous along their depth. In this paper, the two-layer model was chosen for description with account of heterogeneity of a real film.

The task of determination of two-layer system six parameters, refractive indexes, indexes of extinction, and layer thicknesses, is difficult in performing, and its success is limited by ellipsometric function distortion in experimental data limits and by multiplicity of solutions.

That is why it is difficult to do without initial possible assumption concerning the system structure that simplifies procedure of parameters determination. The array of system parameter values that consistently describes the obtained experimental data array is the warranty of assumption fulfillment.

Two versions of the two-layer film can be considered: external layer is optically less dense than the inner layer 

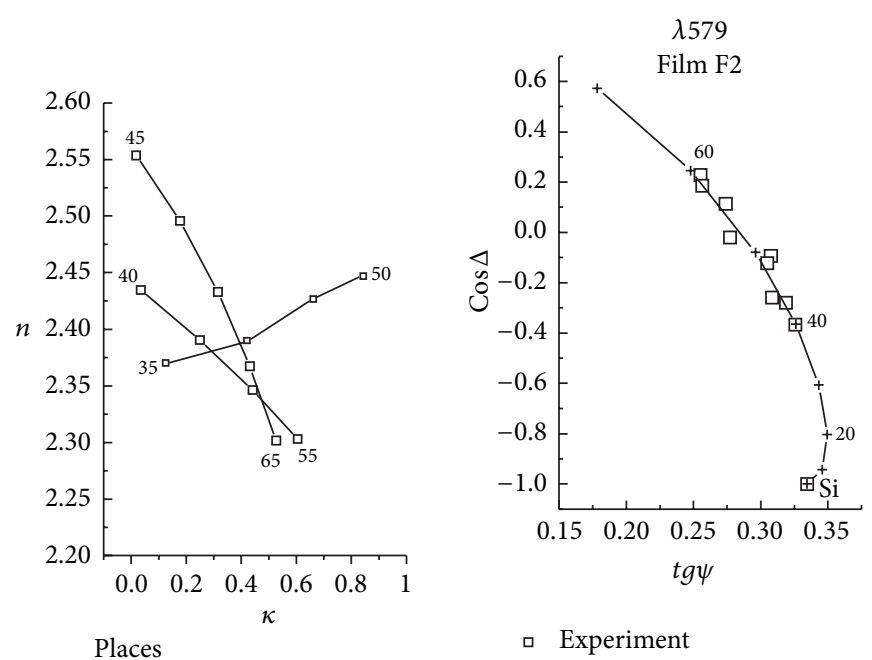

$$
\begin{aligned}
& \text {-ロ- } 1 \\
& -\square-2 \\
& -\square-3
\end{aligned}
$$

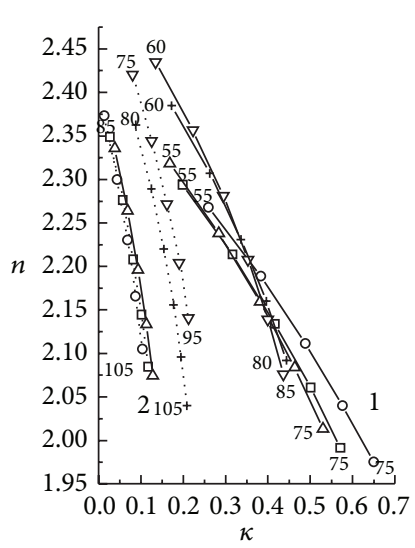

Experiment

-+- Theory

$n_{2}=2.385 \quad \kappa_{2}=0.42$

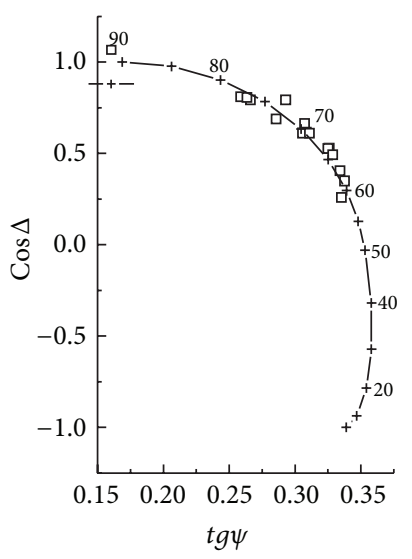

$$
\begin{array}{ll}
\text { Places } & \\
-\square-1 & -\nabla-4 \\
-\circ-2 & -+-5
\end{array}
$$

$-\Delta-3$

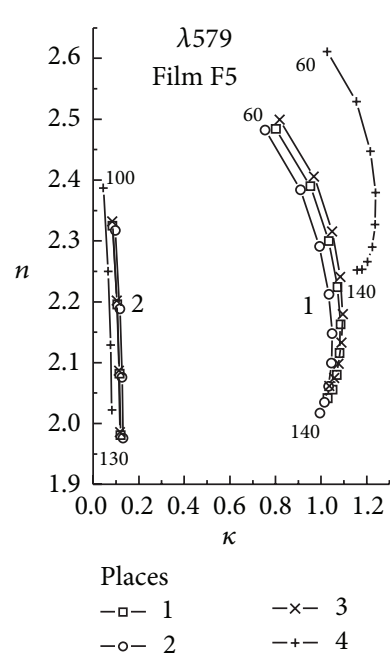

(a)

$$
\begin{aligned}
& \square \quad \text { Experiment } \\
& -+- \text { Theory } \\
& n=2.19 \quad \kappa=0.35
\end{aligned}
$$

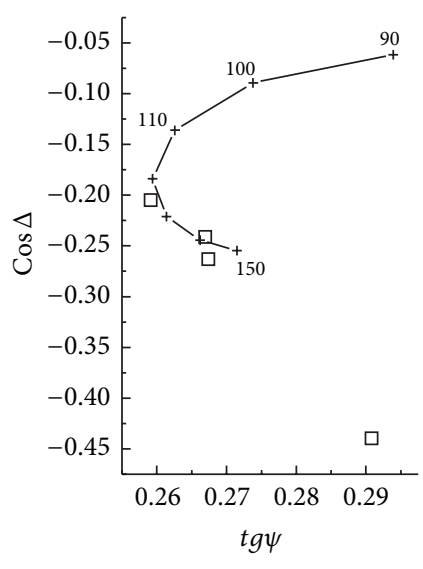

$$
\begin{aligned}
& \square \quad \text { Experiment } \\
& -+- \text { Theory } \\
& n=2.045 \quad \kappa=1.027
\end{aligned}
$$

(b)

FIGURE 2: Range of possible values of refractive index $n$ and index of extinction $\kappa$ (a, curves), calculated for CdTe films on silicon; comparison of experimental data with calculated dependence of ellipsometric parameters $\cos \Delta$ and $\operatorname{tg} \psi$ on the film thickness (b). Numbers near markers specify film thickness in $\mathrm{nm}$. Numbers 1 and 2 indicate solution number. 

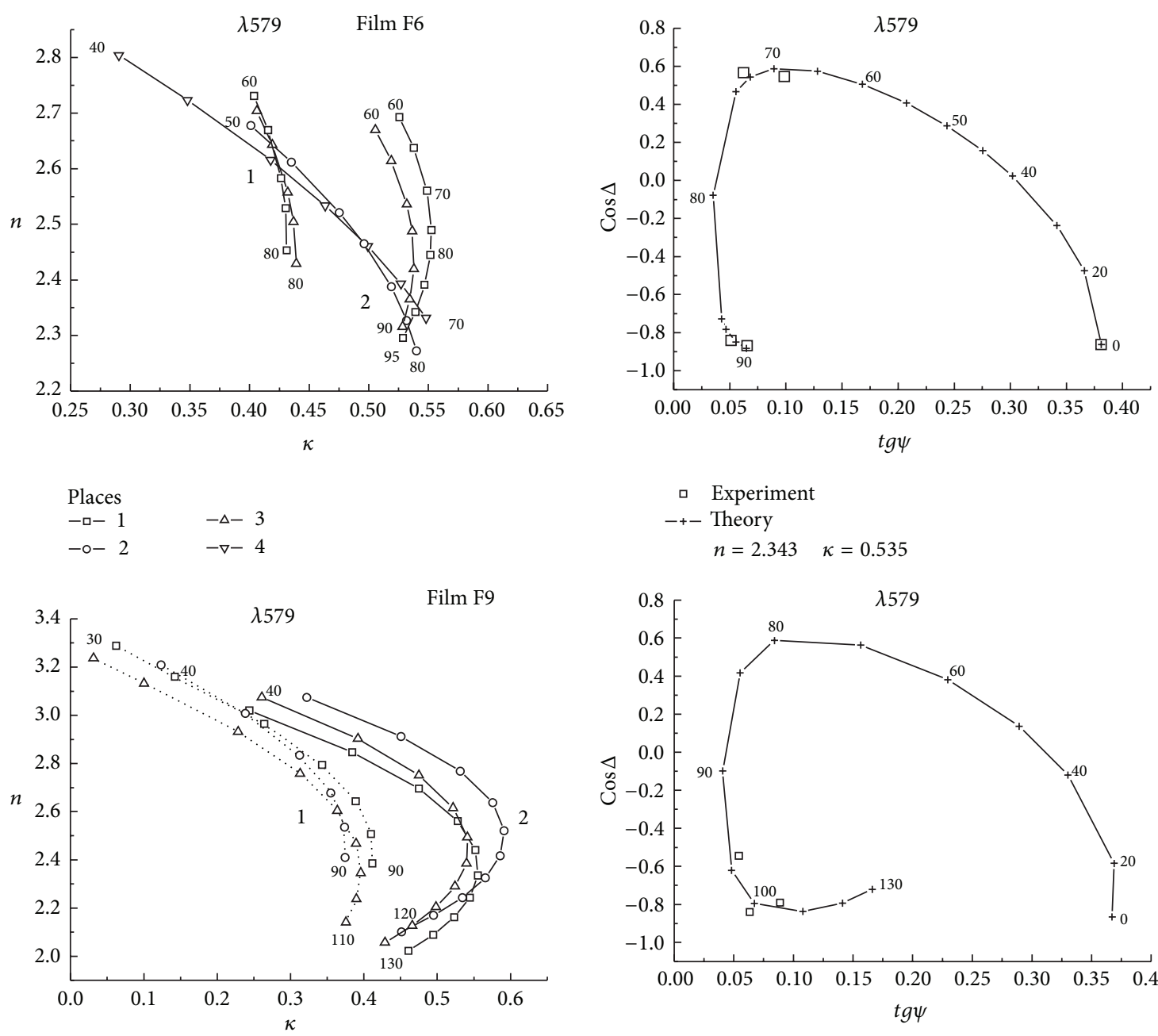

$$
\begin{aligned}
& \text { ㅁ Experiment } \\
& -+- \text { Theory } \\
& n=2.343 \quad \kappa=0.535
\end{aligned}
$$

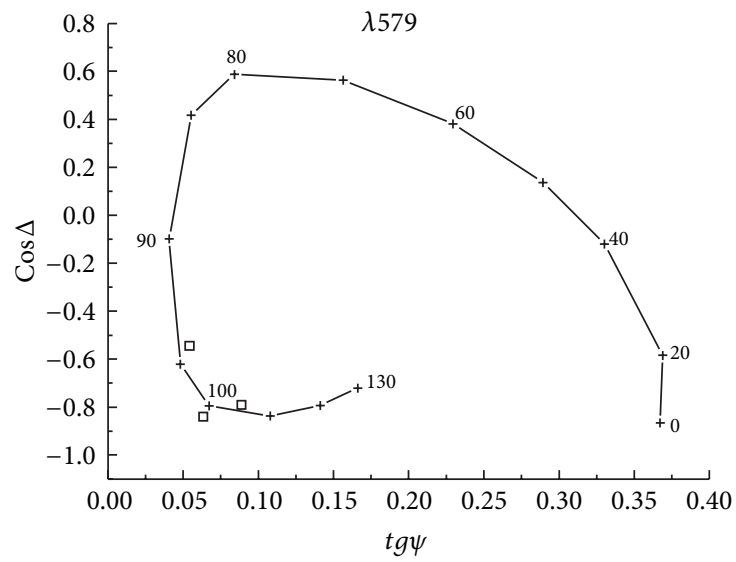

$$
\begin{array}{ll}
\text { Places } & \\
-\square-1 & -\Delta-4
\end{array}
$$

a Experiment

-+- Theory

$$
n=2.29 \kappa=0.537
$$
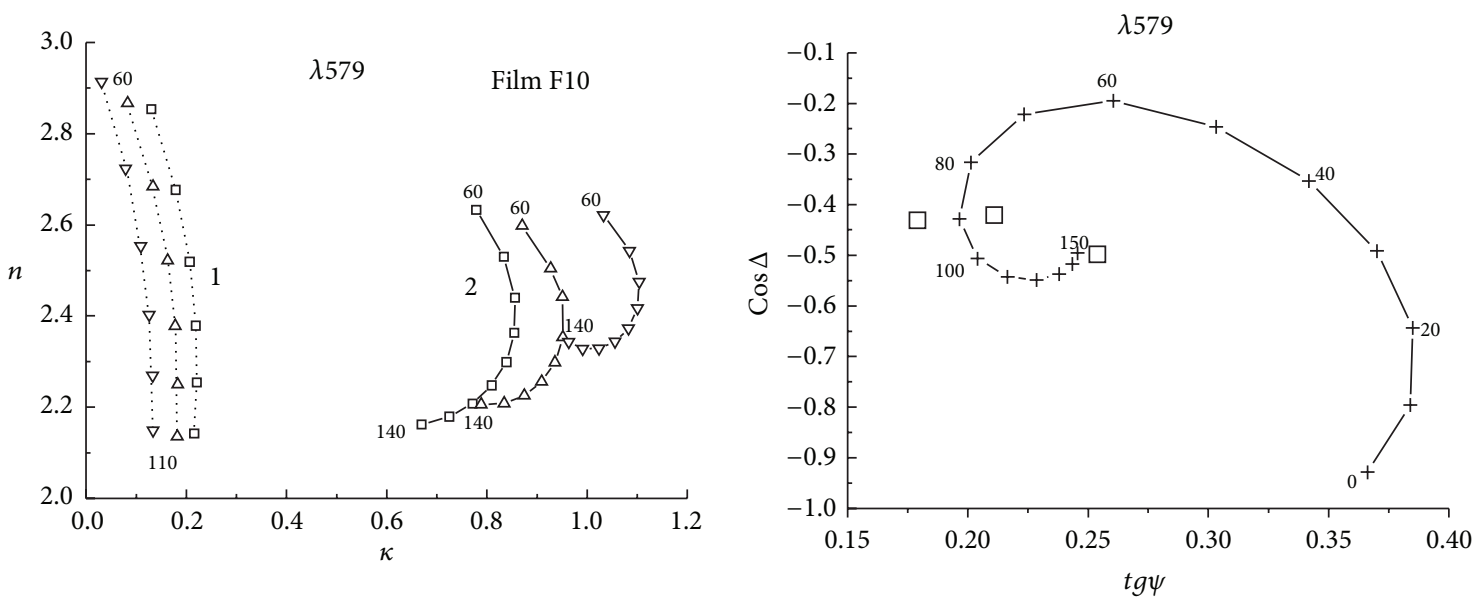

$$
\begin{aligned}
& \text { Places } \\
& -\square-1 \quad-\nabla-3
\end{aligned}
$$

口 Experiment$$
\text { -+- Theory }
$$$$
n=2.361 \quad \kappa=0.907
$$

(a)

(b)

Figure 3: Range of possible values for the refractive index $n$ and index of extinction $\kappa$ (a; curves), calculated for CdTe films on CdHgTe; comparison of experimental data with calculated dependence of ellipsometric parameters $\cos \Delta$ and $\operatorname{tg} \psi$ on film thickness (b). Numbers near markers specify the film thickness in nm. Numbers 1 and 2 indicate solution number. 


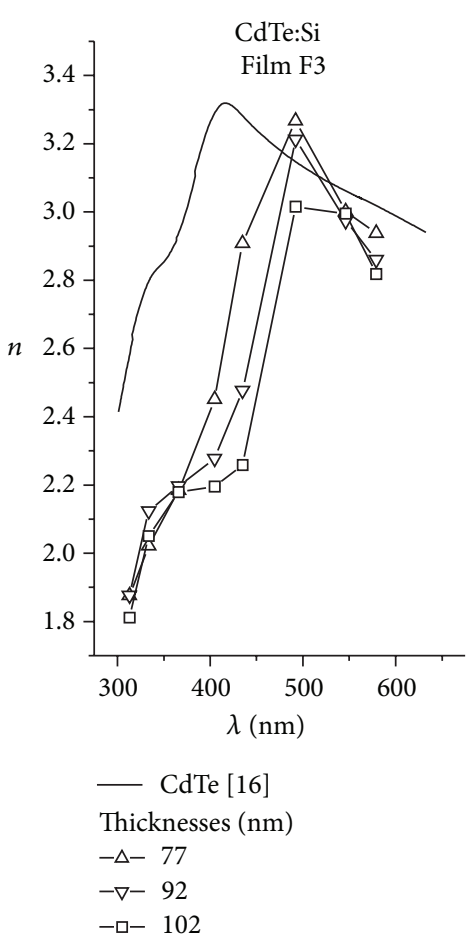

(a)

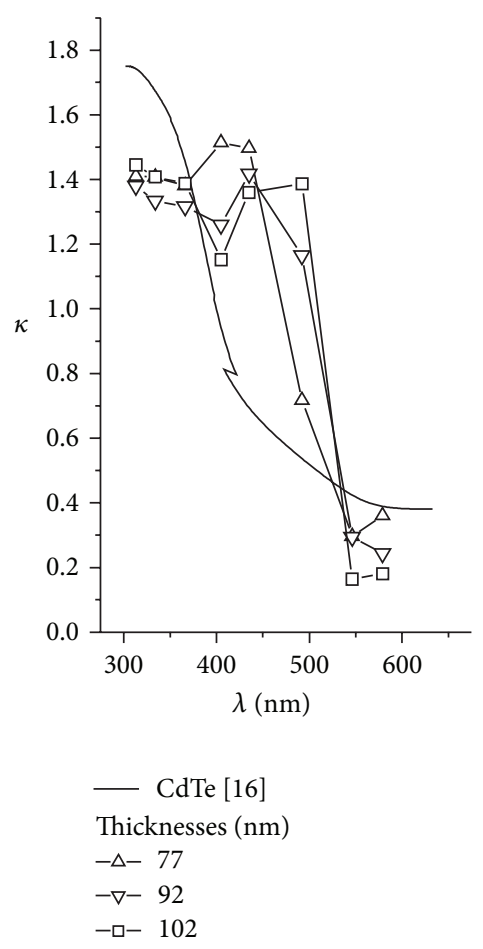

(b)

Figure 4: Optical constants spectra for film F3 depending on the film thickness in different places.

(model 1); vice versa, external layer is optically more dense (model 2).

The model 1 is more probable for real CdTe films; inner layer is a layer from bulk cadmium telluride, and external layer is a mixture from cadmium telluride and cavities completely or partially filled with oxide. Also, it can include film surface roughness. The calculations performed using the model 1 [17] show that best of all it describes experimental data obtained for real CdTe films with rough surface. Contrariwise, the calculations carried out in this paper showed that, when the film structure corresponds to the model 2, the optical spectrum distortions described earlier were not observed.

Therefore, the next task is to find two-layer film parameters taking into account that its structure confirms the model 1.

Optical constants for substrates (Si, CdHgTe) and film matter (CdTe) are known with ample accuracy. Reflective system parameters for determination are refractive index $n_{1}$, index of extinction $\kappa_{1}$ for upper layer, and thickness for upper $d_{1}$ and lower $d_{2}$ layers, four unknown parameters. The problem of four unknown values determination could be solved by the Method of Thickness Curves (MTC) [12].

In this case, algorithm for the task solved using MTC differs from that described in [12] and used earlier for the one-layer film. It consists of consecutive performance of the following steps.

(1) Thickness $d_{1}$ of the upper layer is chosen, for instance, close to an expected value of mean-square roughness height.
(2) Changing $d_{2}$ in defined limits and using the software [13], $n_{1}$ and $\kappa_{1}$ are determined for these thicknesses (thickness curves); experimental values of ellipsometric parameters obtained at light reflection from all measured film places are used. Thus, several value arrays of required system parameters are obtained by the number of work places.

(3) Thickness curves are plotted on the diagram in $n_{1}-\kappa_{1}$ coordinates. Thickness curves intercross in pairs and form the region of relative intercross.

(4) Thickness curves calculation is reiterated by changing $d_{1}$, while region of relative intercross would be the closest.

Ellipsometric data for tested films were recalculated using the two-layer model 1; results of film optical parameters calculation are presented in Table 2 .

Attempt to find parameters of the two-layer system by using the model 2 was inefficient; unreal values of the extinction index (above unity) were obtained.

Several constitutive conclusions could be made from the data presented in Table 2.

(1) Optical constants of the upper layer for all the tested films $\left(n_{1}\right.$ and $\left.\kappa_{1}\right)$ are lower than optical parameters of bulk cadmium telluride (3.04 and 0.29 correspondingly, $\lambda=632 \mathrm{~nm}$ ).

(2) Thickness of these two layers increases both for CdTe:Si and CdTe:CdHgTe films, but this growth is realized with a different rate. Thus, the thickness of 
TABLE 2: Refractive index $n$, index of extinction $\kappa$, and CdTe film thickness $d$, calculated using the one-layer model; refractive index $n_{1}$, index of extinction $\kappa_{1}$, and thickness $d_{1}$ of the outer layer and thickness $d_{2}$ of the inner layer, calculated using the two-layer model $(\lambda=632.8 \mathrm{~nm})$.

\begin{tabular}{|c|c|c|c|c|c|c|c|}
\hline \multirow{2}{*}{ Film number } & \multicolumn{3}{|c|}{ One-layer model } & \multicolumn{4}{|c|}{ Two-layer model } \\
\hline & $n$ & $\kappa$ & $d, \mathrm{~nm}$ & $n_{1}$ & $\kappa_{1}$ & $d_{1}, \mathrm{~nm}$ & $d_{2}, \mathrm{~nm}$ \\
\hline \multicolumn{8}{|c|}{ CdTe films on $\mathrm{Si}$} \\
\hline $\mathrm{F} 1$ & 2.40 & 0.35 & $83-85$ & $2.66-2.69$ & $0.18-0.05$ & $40-60$ & $10-30$ \\
\hline $\mathrm{F} 2$ & 2.32 & 0.456 & $50-60$ & 2.35 & $0.025-0.225$ & $30-40$ & $13-27$ \\
\hline F3 & 2.715 & 0.251 & $80-110$ & 2.8 & 0.19 & 60 & $7-40$ \\
\hline $\mathrm{F} 4$ & 2.36 & 0.222 & $60-70$ & 2.41 & 0.13 & 50 & $6-20$ \\
\hline F5 & 2.8 & 1.25 & $\sim 120$ & 2.275 & 0.025 & 120 & 5 \\
\hline \multicolumn{8}{|c|}{ CdTe films on CdHgTe } \\
\hline F6 & 2.67 & 0.333 & $45-70$ & 2.65 & 0.225 & 35 & $11-27$ \\
\hline F7 & 2.372 & 0.671 & $80-82$ & 2.35 & 0.068 & 70 & $20-30$ \\
\hline F8 & 3 & 0.3 & 80-105 & 2.62 & 0.12 & 100 & $5-25$ \\
\hline F9 & 2.55 & 0.43 & 94-105 & 2.35 & 0.16 & 60 & $35-40$ \\
\hline F10 & 2.8 & 1.1 & $90-140$ & 2.3 & 0.04 & 100 & $15-20$ \\
\hline
\end{tabular}

inner layer that is the layer of hard-grained cadmium telluride does not exceed $40 \mathrm{~nm}$ (samples $\mathrm{F} 3$ and F9). At the same time, the thickness of outer layer can amount $120 \mathrm{~nm}$ (sample F5) or $100 \mathrm{~nm}$ (films F8 and F10). So, for films with the sputtering time $10 \cdots 14$ minutes the majority thickness conforms to the mixture of cadmium telluride and impurities, which decreases both refractive index and index of extinction. It could be suggested that during matter sputtering in reaction chamber formation of the bulk film is the first, which with the lapse of time is replaced by formation of the upper faulted layer of cadmium telluride with impurities.

(3) Films F5 and F10 that were formed during 14 minutes are exceptions. They are mostly faulted; the thickness of bulk cadmium telluride inner layer is $5 \mathrm{~nm}$ (sample F5) and $15 \cdots 20 \mathrm{~nm}$ (sample F10). It is difficult to suggest that during long-term sputtering bulk layer is formed firstly and then this layer is faulted with time. Obviously, some uncontrolled factors have led to formation of the faulted film from the very beginning.

\section{Results and Discussion}

Ellipsometric measurements indicate that films obtained by the vacuum "hot-wall" epitaxy method are heterogeneous. There are two types of heterogeneity, which appear simultaneously: (1) films' heterogeneity over the sample area; (2) films' heterogeneity along its thickness. The second type can indicate discontinuity of gas flow in reactor chamber which is induced by temperature inhomogeneous distribution in it and inhomogeneous process of film sputtering onto the cold substrate $\left(T_{\text {sub }} \approx 50 \cdots 80^{\circ} \mathrm{C}\right)$, which influences the film thickness.

The first type of heterogeneity appears at different film thickness values for different places of its surface. The difference of thicknesses equals $20 \cdots 30 \mathrm{~nm}$.
Heterogeneity along the thickness of films appears as the fact that spectra of optical parameters calculated in frames of the simple one-layer model are distorted. Specifically, Figure 4 shows that characteristic peculiarities of the cadmium telluride spectrum shift to the long-wave region by the value up to $150 \mathrm{~nm}$.

As was ascertained in numerous investigations, optical spectra of cadmium telluride within this spectral range are caused by direct electron transitions from the valence band, split by spin-orbit interaction, to the lower conduction band. These transitions are achieved in the direction $\Lambda$ near the point L of Brillouin zone and form the well-known in literature doublet that is located in the near ultraviolet range of spectrum close to $400 \mathrm{~nm}$. The region of abnormal dispersion in the refractive index spectrum, which corresponds to this doublet, is also located here. All these peculiarities appear in the spectra obtained by us (Figure 4 ).

The reason for changes in the optical spectra could be considerable variation in the energy band structure, which occur in the direction $\Lambda$ near the point $\mathrm{L}$ of Brillouin zone. It is expressed particularly in the fact that with increasing the sputtering time, the minimum of conduction band in the point L lowers at the expense of changes in the film crystalline structure and correspondent energy gaps in the band scheme decrease. It leads to a long-wave shift of the maximum in the observed spectra. Energy structure changes could be caused by distortion of films crystalline structure, which increases with the sputtering time growth and is difficult to be explained.

Though, it is difficult to imagine that thermal field small nonhomogeneity that exists in this technology or other factors of film formation can cause such cardinal rebuilding of the energy band structure inherent to these films.

In our opinion, the obtained data could be explained by films' heterogeneity along their thickness. In particular, this heterogeneity seems possible to be described using the twolayer film model (Section 2.2) where the inner layer is a layer of bulk cadmium telluride. 


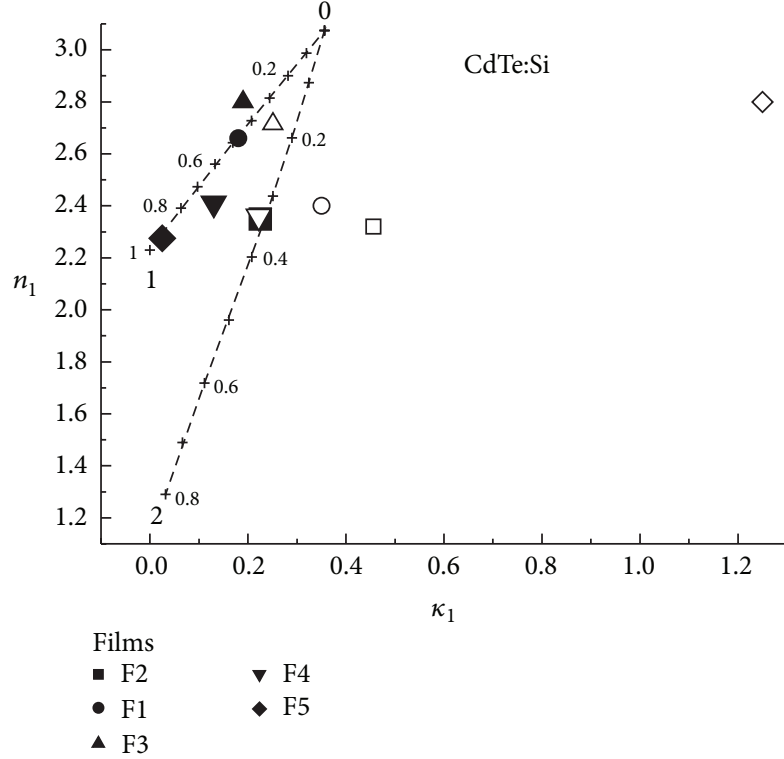

(a)

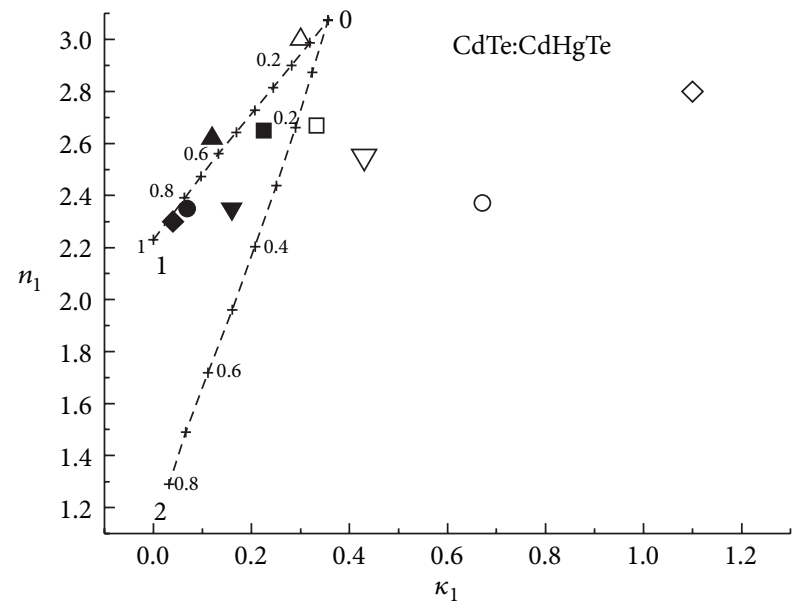

$\begin{array}{ll}\text { Films } & \\ \text { - F6 } & \text { V F9 } \\ \text { - F7 } & \text { • F10 } \\ \text { \ F8 } & \end{array}$

(b)

FiguRE 5: Refractive index $n_{1}$ and index of extinction $\kappa_{1}$ of outer layer of cadmium telluride films (symbols), grown on silicon (a) and CdHgTe (b) substrates. Curves present optical constants' change of the mixtures CdTe + oxide (1) and CdTe + air (2). Numbers near markers specify the volume fraction of oxide and air in mixtures.

Use of the two-layer model for CdTe films is justified using the following reasons. According to atomic force microscopy [11] on surface of CdTe films, obtained by the vacuum "hot-wall" epitaxy method at the same device as the films studied in this paper, roughness at the thicknesses $5 \cdots 100 \mathrm{~nm}$ always exists. The film could be regarded as homogenous with an averaged portion of cavities in it, if the mean-square high of the roughness is much less than the light wavelength [18].

Optical constants of this equivalent film depend on the volume fraction of cavities, filling material, and they could be calculated using the theory of effective medium in Bruggeman or Maxwell-Garnett approximations [19].

No one of these approximations can explain the transformation of the optical constants spectra observed in Section 2.1, specifically that the index of extinction in the long-wave spectral range can appreciably exceed the index of extinction for film matter in the bulk state. In any of the mentioned above approximations, availability of cavities can only decrease the values of optical constants.

At the same time, use of the two-layer film model can explain the ellipsometric data set.

In [17], model calculations show that neglecting the near-surface layer on CdTe film, particularly formed by roughness, in the one-layer film model leads to refractive index decreasing and simultaneous increasing in the index of extinction. Detected in such a way optical constants by their physical meaning are equivalent to optical constants of the one-layer film, which replaces the real two-layer film.

So, use of the two-layer model for CdTe films in some cases is justified and leads to formation of consistent pattern for the reflective system structure of cadmium telluride films.
With the aim to ascertain nature of impurities in the outer layer of CdTe film, calculation of optical parameters for this three-component mixture of cadmium telluride, its oxide, and air was performed.

In Bruggeman approximation [19], mixture components are considered as spherical particles located in effective field formed by all atoms of matter. Dielectric constant $\varepsilon_{\text {eff }}$ of this mixture is described by the following equation:

$$
f_{1} \frac{\varepsilon_{1}-\varepsilon_{\mathrm{eff}}}{2 \varepsilon_{\mathrm{eff}}+\varepsilon_{1}}+f_{2} \frac{\varepsilon_{2}-\varepsilon_{\mathrm{eff}}}{2 \varepsilon_{\mathrm{eff}}+\varepsilon_{2}}=0
$$

where $f_{1}$ and $f_{2}$ are volume fractions of components with dielectric constants $\varepsilon_{1}$ and $\varepsilon_{2}$. The equality $f_{1}+f_{2}=1$ is performed.

First, optical constants for the two-component mixture oxide and air (cavities filler material) were calculated using (1); second, the same calculation was carried out for the mixture of cadmium telluride and filler matter.

The optical constants of CdTe $n=3.04, \kappa=0.29$ [16] $(\lambda=632 \mathrm{~nm})$ and refractive index of the oxide layer $n=2.23$ on cadmium telluride surface [20] were used for computing.

Results for optical constants calculation in the case of three-component medium are presented by two curves in Figure 5. The upper curve 1 represents optical constants of the mixture CdTe + oxide and the curve 2 represents optical constants of the mixture $\mathrm{CdTe}+$ air. The volume fraction of oxide (from 0 to 1 ) and air $(0 \cdots 0.8)$ increases along curves 1 and 2, respectively. The area between upper and lower curves describes optical constants of three-component mixture $\mathrm{CdTe}+$ oxide + air with different volume fractions of mixture components. 
Figure 5 also presents optical constants of surface layer for ten studied films denoted in Table 2 (upper layer) calculated using the one-layer model (empty symbols) and the two-layer one (filled symbols) for the same samples.

Obviously, optical constants for majority films, calculated using the one-layer model, are found in the diagram region that lies outside the range of values corresponding to the cavities filled with air and oxide (area between the curves 1 and 2). Filling the cavities with some conjectural matter with a high index of extinction that exceeds the index of extinction of monocrystalline cadmium telluride ought to be assumed for this fact explanation in frames of the effective medium theory.

At the same time, results of these films calculation by using the two-layer model (filled symbols) are found in the area between the curves 1 and 2, that is, in the area of possible values for optical constants corresponding to the mixture of cadmium telluride, its oxide, and air.

There is no sense to cite detailed data about the volume fraction of impurities in the outer layer of films due to sufficiently large errors in two-layer model calculation. These errors occur as a consequence of small number of places with different thicknesses and at worst can amount to the value $\Delta \kappa= \pm 0.1$. Only qualitative differences between films could be considered with confidence.

Specifically, experimental data for four films from five, grown on silicon substrate (Figure 5(a)), lay on the curve 1; that is, the outer layer is a mixture of cadmium telluride and its oxide, which volume fraction in the mixture changes from 0.4 (film F3) up to 0.9 (film F5).

The outer layer of CdTe films grown on CdHgTe (Figure 5(b)) corresponds to the mixture of cadmium telluride and its oxide for majority films. Only the film F9 is three-component with approximately equal fractions of oxide and air in the cavities filled.

The observed outer layer properties could be fully or partially stipulated by the surface relief, at least its thickness approximates typical values of the mean-square roughness high for cadmium telluride films deposited in the same facility [11]. If so, some conclusions about the shape of relief for films' surface could be made from the Figure 5 data.

Thus, the film F5, with a high oxide fraction $(\sim 0.9)$ and that of a small matter fraction (Figure 5(a)), can have a relief consisting of high fine peaks. These peaks are located at short distances of about $5 \mathrm{~nm}$ from each other (thickness of natural oxide) and are almost completely oxidized.

At the same time, the film F3 with a smaller oxide fraction $(\sim 0.4)$ can have roughness in the shape of flat humps.

In the frames of this study, relation of the filling matter volume fraction with the relief shape is not a theme for detail analysis; relief shape data are needed for it.

Consequently, the ellipsometric data and their treatment permitted to ascertain that all the studied films are the twolayer ones; the upper surface layer is filled with the mixture of cadmium telluride, its oxide, and air, and the inner layer is a layer of hard-grained cadmium telluride.

Though AFM study was not performed for these samples, the films obtained at identical setup [11] always have a relief.
The relief layer and its extension into the bottom layer depth appear ellipsometrically as an equivalent surface layer.

The volume fraction of mixture components was possible to be determined. Increasing the interval of film thicknesses on its area and focusing measurement locality will permit to increase the measurement accuracy for the components' volume fraction.

\section{Conclusions}

Use of the one-layer model for processing the ellipsometric data obtained at light reflection from CdTe films in the case of large thicknesses $>70 \mathrm{~nm}$ can lead to significant distortion of optical constants and their spectra as compared to those of bulk monocrystalline cadmium telluride. It is the consequence of incorrect choosing of the reflective system model.

Real cadmium telluride films have a structure that could be presented within the frame of the two-layer model with an inner layer of bulk CdTe adjacent to the substrate. The outer layer is a layer with optical parameters described by effective-medium approximation; medium consists of three components, cadmium telluride, voids filled with air partially or fully, and oxide.

In general, the film thickness increases with deposition time growth (from 8 to 14 minutes in the given setup). The inner layer can reach the thickness close to $40 \mathrm{~nm}$, and the outer layer thickness becomes larger than that of the inner layer. In some cases, almost all the film is defective and contains the above mentioned mixture.

The outer layer identified ellipsometrically could be represented by a layer with rough surface that is partially or fully oxidized.

\section{Conflict of Interests}

The authors declare that there is no conflict of interests regarding the publication of this paper.

\section{References}

[1] G. K. M. Thutupalli and S. G. Tomlin, "The optical properties of thin films of cadmium and zinc selenides and tellurides," Journal of Physics D: Applied Physics, vol. 9, no. 11, pp. 1639-1646, 1976.

[2] B. R. Mehta, S. Kumar, K. Singh, and K. L. Chopra, "Application of spectroscopic ellipsometry to study the effect of surface treatments on cadmium telluride films," Thin Solid Films, vol. 164, pp. 265-268, 1988.

[3] S. Sirohi, V. Kumar, and T. P. Sharma, "Optical, structural and electrical properties of CdTe sintered films," Optical Materials, vol. 12, no. 1, pp. 121-125, 1999.

[4] Y. Laaziz, A. Bennouna, N. Chahboun, A. Outzourhit, and E. L. Ameziane, "Optical characterization of low optical thickness thin films from transmittance and back reflectance measurements," Thin Solid Films, vol. 372, no. 1-2, pp. 149-155, 2000.

[5] M. Rusu and G. G. Rusu, "On the optical properties of evaporated CdTe thin films," Physics of Low-Dimensional Structures, no. 3-4, pp. 105-115, 2002. 
[6] P. D. Paulson and X. Mathew, "Spectroscopic ellipsometry investigation of optical and interface properties of CdTe films deposited on metal foils," Solar Energy Materials and Solar Cells, vol. 82, no. 1-2, pp. 279-290, 2004.

[7] F. C. Peiris, Z. J. Weber, Y. Chen, and G. Brill, “Optical properties of $\mathrm{CdSe}_{x} \mathrm{Te}_{1-x}$ epitaxial films studied by spectroscopic ellipsometry," Journal of Electronic Materials, vol. 33, no. 6, pp. 724-727, 2004.

[8] V. Odarych, A. Sarsembaeva, F. F. Sizov, and M. Vuychik, "Determination of parameters of cadmium telluride films on silicon by the methods of main angle and multiangular ellipsometry," Semiconductor Physics, Quantum Electronics and Optoelectronics, vol. 8, no. 4, pp. 55-59, 2005.

[9] K. N. Kornienko, V. A. Odarych, L. V. Poperenko, and M. V. Vuichyk, "Determination of optical parameters of CdTe films by principal angle ellypsometry," Functional Materials, vol. 13, no. 1, pp. 179-182, 2006.

[10] E. R. Shaaban, N. Afify, and A. El-Taher, "Effect of film thickness on microstructure parameters and optical constants of CdTe thin films," Journal of Alloys and Compounds, vol. 482, no. 1-2, pp. 400-404, 2009.

[11] Y. Bilevych, A. Soshnikov, L. O. Darchuk et al., "Influence of substrate materials on the properties of CdTe thin films grown by hot-wall epitaxy," Journal of Crystal Growth, vol. 275, no. 1-2, pp. el177-e1181, 2005.

[12] A. Z. Evmenova, V. A. Odarych, F. F. Sizov, and M. V. Vuichyk, "Absorptive CdTe films optical parameters and film thickness determination by the ellipsometric method," Optica Applicata, vol. 38, no. 3, pp. 585-600, 2008.

[13] V. A. Makara, V. A. Odarych, T. Y. Kepich, T. D. Preobrajenskaja, and O. V. Rudenko, "Device and method of measurement of parameters and degree of homogeneity of film structures," Tekhnologiya i Konstruirovanie v Elektronnoi Apparature, vol. 3, no. 81, pp. 40-46, 2009 (Russian).

[14] E. D. Palik, Ed., Handbook of Optical Constants of Solids, Academic Press, New York, NY, USA, 1991.

[15] B. Johs, C. M. Herzinger, J. H. Dinan, A. Cornfeld, and J. D. Benson, "Development of a parametric optical constant model for $\mathrm{Hg}_{1-x} \mathrm{Cd}_{x} \mathrm{Te}$ for control of composition by spectroscopic ellipsometry during MBE growth," Thin Solid Films, vol. 313-314, pp. 137-142, 1998.

[16] S. Adachi, T. Kimura, and N. Suzuki, "Optical properties of CdTe: experiment and modeling," Journal of Applied Physics, vol. 74, no. 5, pp. 3435-3441, 1993.

[17] V. A. Odarych, "The effects of roughness on CdTe films parameters, determined from ellipsometric data," Opticheskii Zhurnal, vol. 7, no. 82, 2015 (Russian).

[18] R. M. A. Azzam and N. M. Bashara, Ellypsometry and Polarized Light, North-Holland, Amsterdam, The Netherlands, 1977.

[19] L. A. Golovan, V. Y. Tymoshenko, and P. K. Kashkarov, "Optical properties of porous-system-based nanocomposites," Uspekhi Phisicheskih Nauk, vol. 177, no. 6, pp. 619-638, 2007.

[20] V. A. Odarich, A. Z. Evmenova, and F. F. Sizov, "Ellipsometric studies of the natural oxide film on the surface of cadmium telluride," Journal of Optical Technology, vol. 80, no. 8, pp. 515519, 2013. 

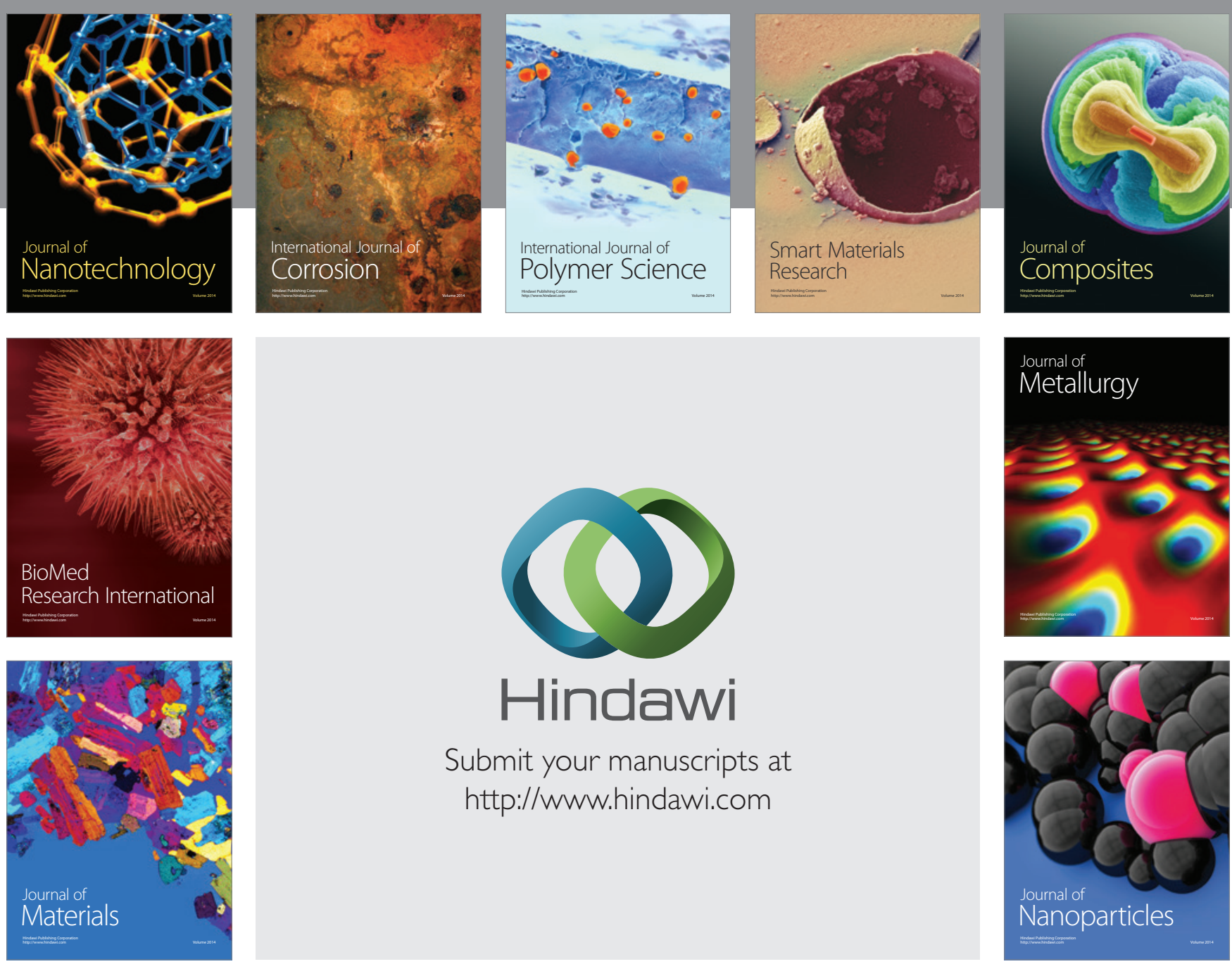

Submit your manuscripts at http://www.hindawi.com
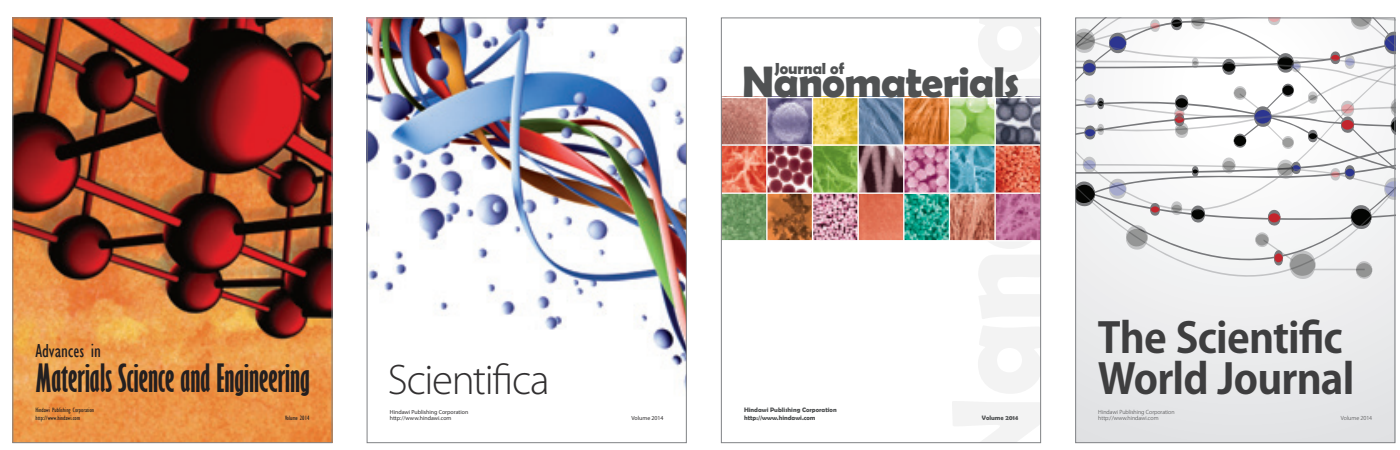

\section{The Scientific World Journal}
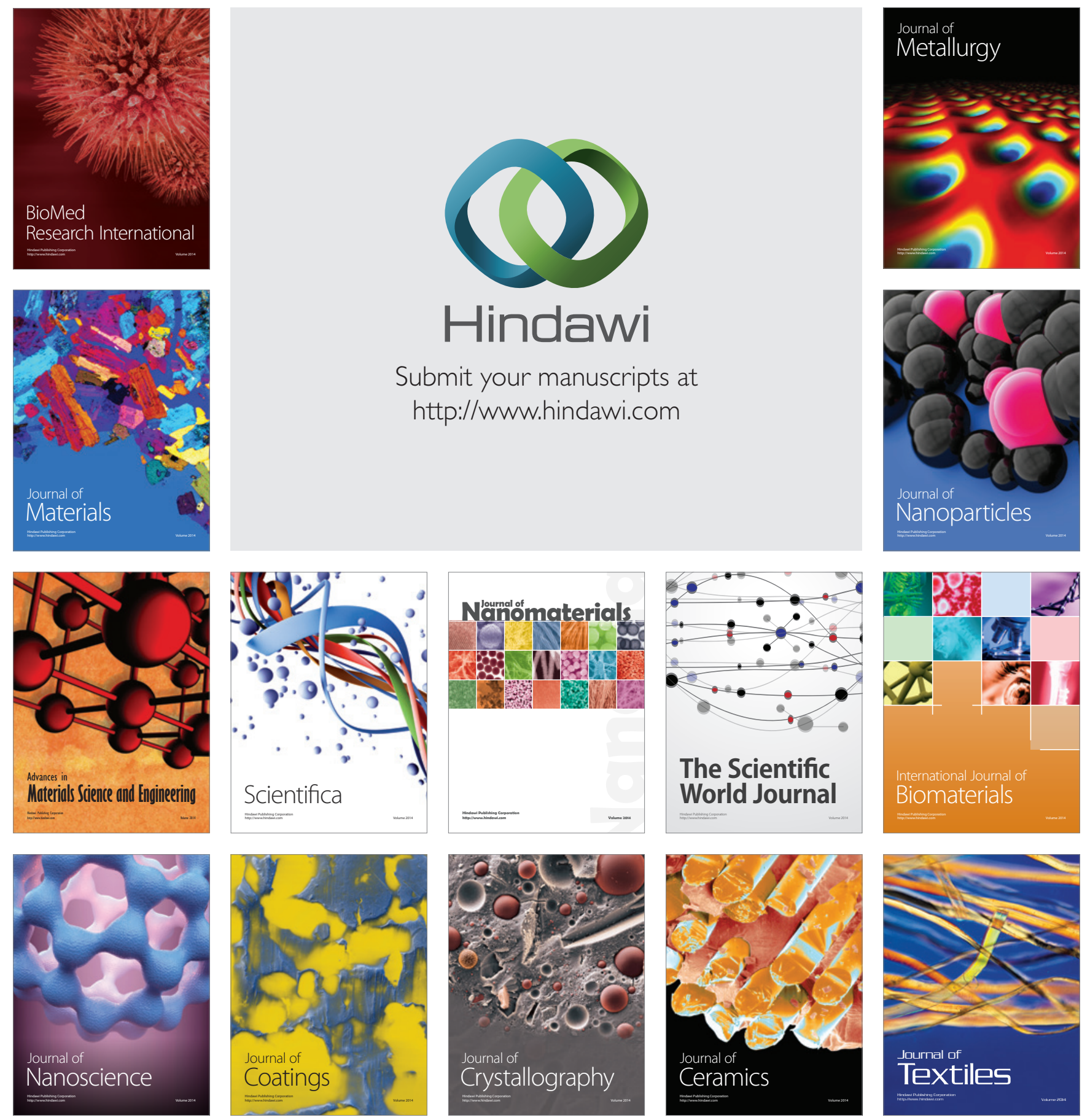